\title{
VARIEDADES DE SORGO SACARINO EM DIFERENTES ESPAÇAMENTOS E POPULAÇÃO DE PLANTAS
}

\author{
ANDRÉ MAY ${ }^{1}$, MÔNICA MATOSO CAMPANHA ${ }^{1}$, ALEXANDRE FERREIRA DA SILVA ${ }^{1}$, \\ MAURÍCIO ANTÔNIO DE OLIVEIRA COELHO², RAFAEL AUGUSTO DA COSTA PARRELLA ${ }^{1}$, \\ ROBERT EUGENE SCHAFFERT ${ }^{1}$ e ISRAEL ALEXANDRE PEREIRA FILHO ${ }^{1}$
}

IEmbrapa Milho e Sorgo, Sete Lagoas, MG, Brasil,andre.may@embrapa.br,monica.matoso@embrapa.br,
alexandre.ferreira@embrapa.br, rafael.parrella@embrapa.br,robert.saffert@embrapa.br, israel.pereira@embrapa.br
${ }^{2}$ Epamig,Fazenda ExperimentalSertãozinho,Patosde Minas,.MG,Brasil,mauricio@epamig.br

Revista Brasileira de Milho e Sorgo, v.11, n.3, p. 278-290, 2012

\begin{abstract}
RESUMO - O sorgo sacarino surgiu como importante alternativa para a geração de biomassa na produção de etanol. Este trabalho objetivou avaliar o efeito do espaçamento entrelinhas e da população de plantas sobre a produção de sorgo sacarino em diferentes locais. Foram instalados dois experimentos, em duas localidades, Patos de Minas, MG e Sete Lagoas, MG, utilizando as cultivares BRS 505 e CMSXS 647, respectivamente. O delineamento experimental utilizado foi em blocos ao acaso, em esquema fatorial 4 x 4, sendo quatro espaçamentos entrelinhas $(0,5 ; 0,6 ; 0,7$ e $0,8 \mathrm{~m})$ e quatro populações de plantas $\left(80.000 ; 100.000 ; 120.000\right.$ e 140.000 plantas ha $\left.^{-1}\right)$. As características avaliadas na colheita foram: altura da planta; diâmetro do colmo; números de perfilhos e de folhas; massa fresca total, de folhas, de colmos e de panículas; massa de caldo; e Brix. O arranjo de plantas influencia o crescimento do sorgo sacarino, resultando em maiores produtividades de caldo em área cultivada com menores espaçamentos entrelinhas e maiores populações de plantas. A redução no espaçamento proporciona maiores incrementos na produção em comparação à elevação na população de plantas, além de aumentar o Brix no caldo.
\end{abstract}

Palavras-chave: Sorghum bicolor, bioenergia, sistemas de produção.

\section{VARIETIES OF SWEET SORGHUM IN DIFFERENT ROW SPACING AND PLANT POPULATION}

\begin{abstract}
The sweet sorghum has emerged as an important alternative to the generation of biomass ethanol. This study evaluated the effect of row spacing and plant population on the production of sweet sorghum. Two experiments were established in two locations, Patos de Minas, MG and Sete Lagoas, MG, using the cultivars BRS 505 and CMSXS 647 , respectively. A randomized blocks design in a 4 x 4 factorial scheme was used, with 4 row-spacings $(0.5,0.6,0.7$ and $0.8 \mathrm{~m})$ and 4 plant populations $\left(80,000 ; 100,000,120,000\right.$ and 140,000 plants $\left.\mathrm{ha}^{-1}\right)$. The characteristics evaluated at harvest were plant height; stem diameter; number of tillers and leaves; fresh mass (total, of leaves, stems and panicles); mass of juice and Brix. The arrangement of plants affected growth of sweet sorghum, resulting in higher yields of juice in an area cultivated with smaller row spacing and larger plant populations. The reduction in spacing provides greater increases in production compared to the increase in plant population, besides increasing the Brix of the broth.
\end{abstract}

Key words: Sorghum bicolor, bioenergy, production systems. 
A demanda mundial por combustíveis de fontes renováveis tem se expandido rapidamente nos últimos anos devido à preocupação com a redução do volume de emissões de gases causadores do efeito estufa, derivados da utilização de combustíveis fósseis. Além disso, incertezas a respeito da disponibilidade futura de recursos não renováveis e tensões geopolíticas em regiões produtoras de petróleo têm despertado grande interesse no mundo pelos biocombustíveis, pois estes são os mais viáveis substitutos para o petróleo em escala significativa.

Dentre as culturas com potencial bioenergético, a cultura da cana-de-açúcar (Saccharum officinarum L.) destaca-se no cenário nacional, com área plantada de aproximadamente 8,4 milhões de hectares, com produção estimada em 22.857,6 bilhões de litros de etanol (Conab, 2011). Em virtude da elevada demanda atual por etanol, o cultivo de sorgo sacarino [Sorghum bicolor (L.) Moench.] surge como importante alternativa para a geração de biomassa para a produção de etanol (Zhao et al., 2009; Zegada-Lizarazu \& Monti, 2012; Han et al., 2012; Ratnavathi et al., 2011), principalmente na entressafra da cana-de-açúcar.

O sorgo sacarino se assemelha à cana-de-açúcar no armazenamento de açúcares nos colmos, os quais possibilitam a produção de etanol, e no fornecimento de quantidade de bagaço suficiente para geração de vapor na operação industrial (Teixeira et al., 1997; Almodares \& Hadi, 2009). Entretanto, ele difere de maneira acentuada da cana-de-açúcar no cultivo a partir de sementes e no ciclo vegetativo bem mais curto, de 120 a 130 dias. Além de atuar como cultura complementar à cana-de-açúcar, possibilitando a colheita da cana após a maturação completa, o que representa teores mais elevados de açúcares, destaca-se por apresentar maior tolerância ao alumínio tóxico no solo, ao déficit hídrico e à salinidade, possi- bilitando seu cultivo em áreas consideradas marginais à agricultura (Amaducci et al., 2004; Vasilakologlou et al., 2011; Prasad et al., 2007). Portanto, o sorgo sacarino apresenta-se como uma interessante opção complementar à cana-de-açúcar para compor a matriz energética nacional, possibilitando a expansão da área passível de utilização para produção de bioenergia e aumentando a eficiência da produção de etanol.

São poucos os trabalhos relacionados com o manejo cultural do sorgo sacarino, principalmente no Brasil (Rodrigues \& Leite, 1999; Albuquerque et al., 2010; Souza et al.; 2011, Teixeira et al., 1999). Cowley \& Smith (1972) verificaram que os rendimentos obtidos na cultura do sorgo sacarino são diretamente influenciados pelo comprimento dos dias e pela radiação solar. Os melhores rendimentos em açúcares por área de cultivo estão estreitamente relacionados com dias mais longos e radiação solar máxima. Então, uma forma de aumentar a interceptação da radiação solar é a escolha adequada do arranjo de plantas. Este arranjo pode ser manipulado pela mudança na densidade de plantas e no espaçamento entrelinhas.

Ótimos rendimentos de biomassa em sorgo sacarino são influenciados pelo manejo, sendo, em geral, também dependentes das condições específicas do local de cultivo. Trabalho conduzido por Albuquerque et al. (2010), envolvendo diferentes arranjos de plantas e duas cultivares, verificou que a redução do espaçamento entrelinhas e o aumento da densidade de semeadura resultaram em maior produtividade de massa verde. Densidade de semeadura e espaçamento entrelinhas influenciando na produtividade também foram verificados por outros autores (Zegada-Lizarazu \& Monti, 2012; Snider et al., 2012). Zhao et al. (2009) encontraram efeito da cultivar e do ano de plantio na produção de matéria seca e no teor de açúcar dos colmos. Em experimentos de Vasilakologlou 
et al. (2011), cultivar, ano e suprimento de água via irrigação influenciaram, entre outros, a produção de matéria fresca, de caldo e Brix de cultivares de sorgo sacarino. Han et al. (2012) encontraram, como resultados, densidade de plantas interagindo com diferentes variáveis ambientais na resposta ao crescimento do sorgo.

Em busca de aumentar o conhecimento em relação ao arranjo de plantas para a máxima produtividade de biomassa, o objetivo deste trabalho foi avaliar o efeito do espaçamento entrelinhas e da população de plantas sobre a produção de diferentes cultivares de sorgo sacarino em dois locais de Minas Gerais: região Central e Alto Paranaíba.

\section{Material e Métodos}

Os experimentos foram instalados em dois locais no estado de Minas Gerais, Patos de Minas (região do Alto Paranaíba) e Sete Lagoas (região Central do estado), com plantio em março de 2011 em cada localidade. As médias da temperatura máxima e mínima e a precipitação acumulada no período experimental foram $26,3{ }^{\circ} \mathrm{C}, 15,4{ }^{\circ} \mathrm{C}$ e $560,9 \mathrm{~mm}$, respectivamente, para Patos de Minas, e $28,5^{\circ} \mathrm{C}, 16,5^{\circ} \mathrm{C}$ e 21,6 mm para Sete Lagoas, respectivamente.

O ensaio em Patos de Minas foi instalado na fazenda experimental da Epamig, situada entre a latitude $18^{\circ} 31^{\prime} \mathrm{S}$ e a longitude $46^{\circ} 26^{\prime} \mathrm{W}$, com altitude média de $929 \mathrm{~m}$. O clima da região é do tipo
AW - Tropical de altitude, segundo classificação de Köppen, com verão quente e seco e inverno frio. $\mathrm{O}$ ensaio em Sete Lagoas foi instalado no campo experimental da Embrapa Milho e Sorgo, situada entre a latitude $19^{\circ} 23^{\prime} \mathrm{S}$ e a longitude $44^{\circ} 10^{\prime} \mathrm{W}$ com altitude média de $726 \mathrm{~m}$. O clima da região é do tipo CWA - Tropical, com chuvas concentradas no verão e um período seco bem definido durante o inverno. $\mathrm{O}$ solo, na área experimental em Sete Lagoas, é do tipo Latossolo Vermelho Distrófico, textura argilosa. Em Patos de Minas, o solo é do tipo Latossolo Vermelho Distroférrico, textura argilosa. A Tabela 1 apresenta a análise de solo feita das áreas experimentais.

O delineamento experimental utilizado para os dois experimentos foi em blocos ao acaso, em esquema fatorial $4 \times 4$, sendo quatro espaçamentos de entrelinhas $(0,5 ; 0,6 ; 0,7$ e $0,8 \mathrm{~m})$ e quatro populações de plantas $(80.000 ; 100.000 ; 120.000$ e 140.000 plantas $h^{-1}$ ), sendo utilizadas três repetições para o experimento de Patos de Minas, MG (Experimento 1) e quatro repetições para o experimento de Sete Lagoas, $\mathrm{MG}$ (Experimento 2). A parcela experimental, nos dois ensaios, foi constituída por quatro linhas de $5 \mathrm{~m}$ de comprimento, sendo as duas linhas centrais consideradas parcelas úteis, tendo como bordadura 0,5 m nas extremidades de cada linha central. Em Patos de Minas, foi utilizada a cultivar BRS 505 e, em Sete Lagoas, a cultivar CMSXS 647, ambas da Embrapa, caracterizadas por possuírem ciclos de 120 dias e média sensibilidade ao fotoperíodo.

TABELA 1. Aspectos químicos dos solos das áreas experimentais em Patos de Minas e Sete Lagoas, na profundidade de $0-20 \mathrm{~cm}$.

\begin{tabular}{|c|c|c|c|c|c|c|c|c|c|c|c|c|}
\hline \multirow{2}{*}{ Local } & $\mathrm{pH}$ & $\mathrm{H}+\mathrm{Al}$ & $\mathrm{Al}$ & $\mathrm{Ca}$ & $\mathrm{Mg}$ & SB & CTC & $\mathrm{K}$ & $P$ & MO & $\mathrm{V}$ & $\mathrm{m}$ \\
\hline & \multicolumn{8}{|c|}{ cmolc dm ${ }^{-3}$} & \multicolumn{2}{|c|}{ dag $\mathrm{dm}^{-3}$} & \multicolumn{2}{|c|}{$\%$} \\
\hline Patos de Minas & 5,9 & 7,0 & 0,20 & 3,4 & 1,2 & 4,9 & 11,9 & 120 & 11,7 & 5,3 & 41,2 & 3,9 \\
\hline Sete Lagoas & 6,4 & 5,38 & 0,02 & 4,3 & 0,80 & 5,3 & 10,7 & 107 & 32,9 & 2,70 & 50,0 & 0,34 \\
\hline
\end{tabular}


Para o preparo de solo, foram realizadas uma aração e duas gradagens antes da instalação dos experimentos em cada local de cultivo. A calagem foi feita para elevar a saturação de bases para $60 \%$, antes da aração, permanecendo o solo em descanso por três meses após a incorporação do calcário. Foram aplicados $120 \mathrm{~kg} \mathrm{ha}^{-1}$ de $\mathrm{N}$ na forma de ureia, $90 \mathrm{~kg} \mathrm{ha}^{-1}$ de $\mathrm{P}_{2} \mathrm{O}_{5}$ na forma de super fosfato simples e $180 \mathrm{~kg}$ $\mathrm{ha}^{-1}$ de $\mathrm{K}_{2} 0$ na forma de cloreto de potássio em $\mathrm{Pa}$ tos de Minas; e $120 \mathrm{~kg} \mathrm{ha}^{-1}$ de $\mathrm{N}$ na forma de ureia, $30 \mathrm{~kg} \mathrm{ha}^{-1}$ de $\mathrm{P}_{2} \mathrm{O}_{5}$ na forma de super fosfato simples e $60 \mathrm{~kg} \mathrm{ha}^{-1}$ de $\mathrm{K}_{2} 0$ na forma de cloreto de potássio em Sete Lagoas, segundo a recomendação para sorgo forrageiro descrita por Alvarez et al. (1999). A dose recomendada de cada nutriente aplicada por metro linear foi calculada para ser distribuída em cada linha de cultivo, conforme o espaçamento de entrelinhas estudado, considerando a mesma dose de nutrientes recomendada por hectare para todos os tratamentos estudados.

Para o controle de plantas daninhas, foi utilizado, em pré-emergência, o herbicida atrazine na dose de 2,51 ha-1. A cultura foi irrigada semanalmente por aspersão convencional, com lâmina de $10 \mathrm{~mm}$. Em Patos de Minas, a irrigação se deu em função da precipitação no período.

A demarcação dos espaçamentos foi feita utilizando um sulcador regulável para cada tratamento, acoplado ao sistema hidráulico de um trator. Após a abertura dos sulcos de $4 \mathrm{~cm}$ de profundidade, foi feita a semeadura manual do sorgo sacarino com o auxílio de uma régua previamente marcada, indicando as distâncias entre as plantas na linha de cultivo para cada população de plantas estudada. Foram semeadas de três a quatro sementes por demarcação da régua e, 15 dias após a semeadura, foi feito o raleio das plantas em cada ponto de semeio, deixando apenas uma plan- ta em cada ponto, de forma a estabelecer a distância ideal entre as plantas para cada população estudada, segundo o espaçamento entrelinhas relacionado a cada tratamento.

Os parâmetros avaliados no dia da colheita nos dois locais de cultivo foram: altura da planta (distância da superfície do solo até a ponta da panícula); diâmetro do colmo (no terço médio das plantas); número de perfilhos; massa fresca total (colmo e folhas); massa de caldo; e sólidos solúveis totais ( ${ }^{\circ}$ Brix). Além dos parâmetros acima referenciados, devido à maior disponibilidade de mão-de-obra no local de cultivo do Experimento 2, realizado em Sete Lagoas/MG, foram ainda mensurados: número de folhas; massa fresca de folhas; massa fresca de colmos; e massa fresca de panículas.

A colheita da biomassa fresca foi realizada quando os grãos das panículas atingiram o ponto farináceo. Para a extração do caldo da massa fresca da planta toda (Experimento 1) e da massa fresca do colmo (Experimento 2), foi retirada uma amostra composta por dez plantas da área útil de cada parcela, após as mensurações das características supracitadas. As plantas foram moídas em uma ensiladeira estacionária e homogeneizadas em uma betoneira acoplada ao equipamento de moagem, sendo uma massa equivalente a $500 \mathrm{~g}$ de cada material triturado e homogeneizado, prensada em uma prensa hidráulica Hidraseme, modelo PHS 250, por 60 seg, com pressão de trabalho de 145 bar pressão sobre a amostra de 250 $\mathrm{Kgf} \mathrm{cm}^{-2}$, resultando em força de prensagem de $45 \mathrm{t}$. Após prensagem, o caldo foi pesado, sendo o seu Brix mensurado com refratômetro, logo após a extração da biomassa fresca.

As variáveis de massa fresca total e massa de caldo de cada parcela foram convertidas em produtividade, toneladas por hectare. Os dados obtidos em cada 
experimento foram submetidos à análise de variância e, em caso de significância, os valores foram submetidos ao teste Tukey de médias e a análise de regressão. Todas as análises foram realizadas utilizando o programa estatístico Estat, pertencente à Unesp, Campus Jaboticabal, SP.

\section{Resultados e Discussão}

\section{Experimento I - Patos Minas, MG}

Os resultados da análise de variância do experimento conduzido em Patos de Minas, MG, utilizando a cultivar BRS 505, demonstraram que houve interação altamente significativa entre espaçamento e população de plantas para a produtividade de massa fresca total e massa do caldo. Foi observado efeito isolado altamente significativo da população de plantas para o diâmetro do colmo e Brix do caldo e, também, do espaçamento para o número de perfilhos e Brix do caldo. Porém, não houve efeito significativo dos fatores estudados para altura de planta, sendo a altura média de 2,34 $\mathrm{m}$.

A produtividade de massa fresca total variou em função do espaçamento e da população de plantas. As maiores produtividades, de $76,93 \mathrm{t} \mathrm{ha}^{-1}, 80,61 \mathrm{t}$ $\mathrm{ha}^{-1}$ e 75,24 tha ${ }^{-1}$, nos estandes de $100 \mathrm{mil}, 120$ mil e 140 mil plantas ha $^{-1}$, respectivamente, foram encontradas no menor espaçamento entrelinhas (0,5 m). Já para a população de 80 mil plantas ha1, observou-se a maior produtividade, de $66 \mathrm{t} \mathrm{ha}^{-1}$, no espaçamento de $0,64 \mathrm{~m}$ entrelinhas, segundo o modelo de regressão ajustado (Figura 1).

Avaliando o rendimento de massa fresca total da cultivar BRS 505, com população de plantas ao redor de 120.000 plantas por hectare e espaçamentos entrelinhas de $0,7 \mathrm{~m}$, Marchezan \& Silva (1984) observaram 46,8 t ha-1 em Santa Maria, RS, com semeio em novembro de 1982, e Teixeira et al. (1999), estudando a mesma cultivar por três safras consecutivas a partir do ano de 1984, em Jundiaí, SP, encontraram 20,8, 38,9 e $52,7 \mathrm{t} \mathrm{ha}^{-1}$, respectivamente, para cada ano, sendo menores com semeios tardios (janeiro do último ano de estudo) comparativamente aos demais anos, com semeios realizados em dezembro dos dois primeiros anos.

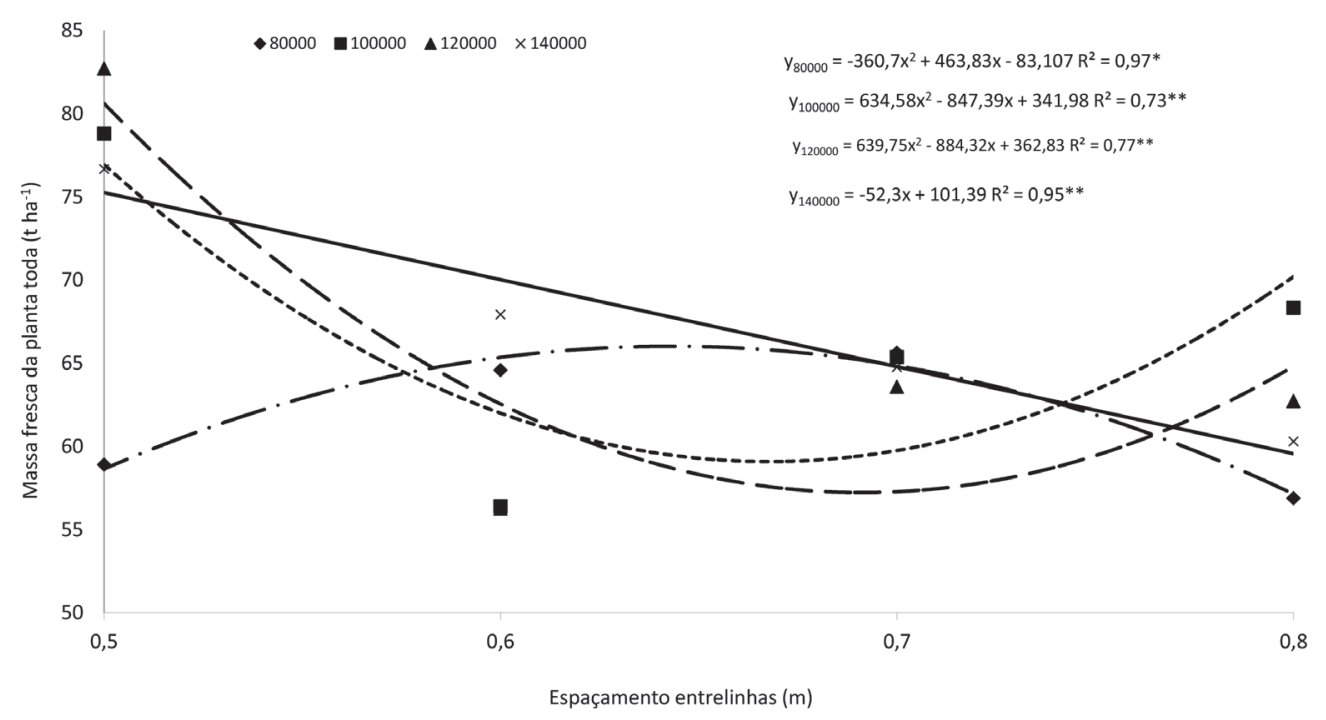

FIGURA 1. Massa fresca total da cultivar de sorgo BRS 505 (Sorghum bicolor), plantada em Patos de Minas, MG, em função do espaçamento entrelinhas. 
Analisando as diferentes densidades de semeadura nos espaçamentos, observou-se comportamento diferenciado entre os stands, com exceção da distância de $0,7 \mathrm{~m}$ na entrelinha, em que não houve diferença significativa na produção de massa fresca total (Figura 1). Albuquerque et al. (2010), estudando diferenças de espaçamento na produção de matéria verde em cultivares BRS 506 e 507 de sorgo sacarino no Semiárido mineiro, encontraram pouca ou nenhuma diferença significativa na produção de matéria verde das cultivares em função do espaçamento, sendo que todas as produtividades foram menores do que aquelas encontradas em Patos de Minas. Os rendimentos de massa verde obtidos neste trabalho estão dentro da amplitude de rendimento de massa verde (27,9 a 124 $\mathrm{t} \mathrm{ha}^{-1}$ ) encontrada por Almodares \& Hadi (2009) em 36 materiais de sorgo sacarino.
A produtividade de massa de caldo apresentou o mesmo comportamento que a produtividade de massa fresca total. Os maiores rendimentos de caldo foram obtidos no menor espaçamento $(0,5 \mathrm{~m})$, sendo de 47,05 tha-1 45,17 tha $\mathrm{th}^{-1}$ e 46,84 $\mathrm{tha}^{-1}$ nos estandes de 140 mil, 120 mil e 100 mil plantas ha ${ }^{-1}$, respectivamente, exceto para a população de 80.000 plantas $\mathrm{ha}^{-1}$, que apresentou o menor rendimento neste espaçamento, de 33,88 tha-1 (Figura 2). Não houve diferença entre os tratamentos na distância de $0,7 \mathrm{~m}$ entre as fileiras.

Houve redução no diâmetro de colmo em função do aumento da população de plantas. $\mathrm{O}$ aumento da população de plantas de 80.000 para 140.000 plantas ha ${ }^{-1}$ ocasionou decréscimo de $2,4 \mathrm{~mm}$ no diâmetro do colmo, passando de $16,48 \mathrm{~mm}$ para $14,08 \mathrm{~mm}$, de acordo com a equação $\mathrm{Y}=4 \mathrm{E}-05 \mathrm{X}+19,683\left[\mathrm{R}^{2}=\right.$

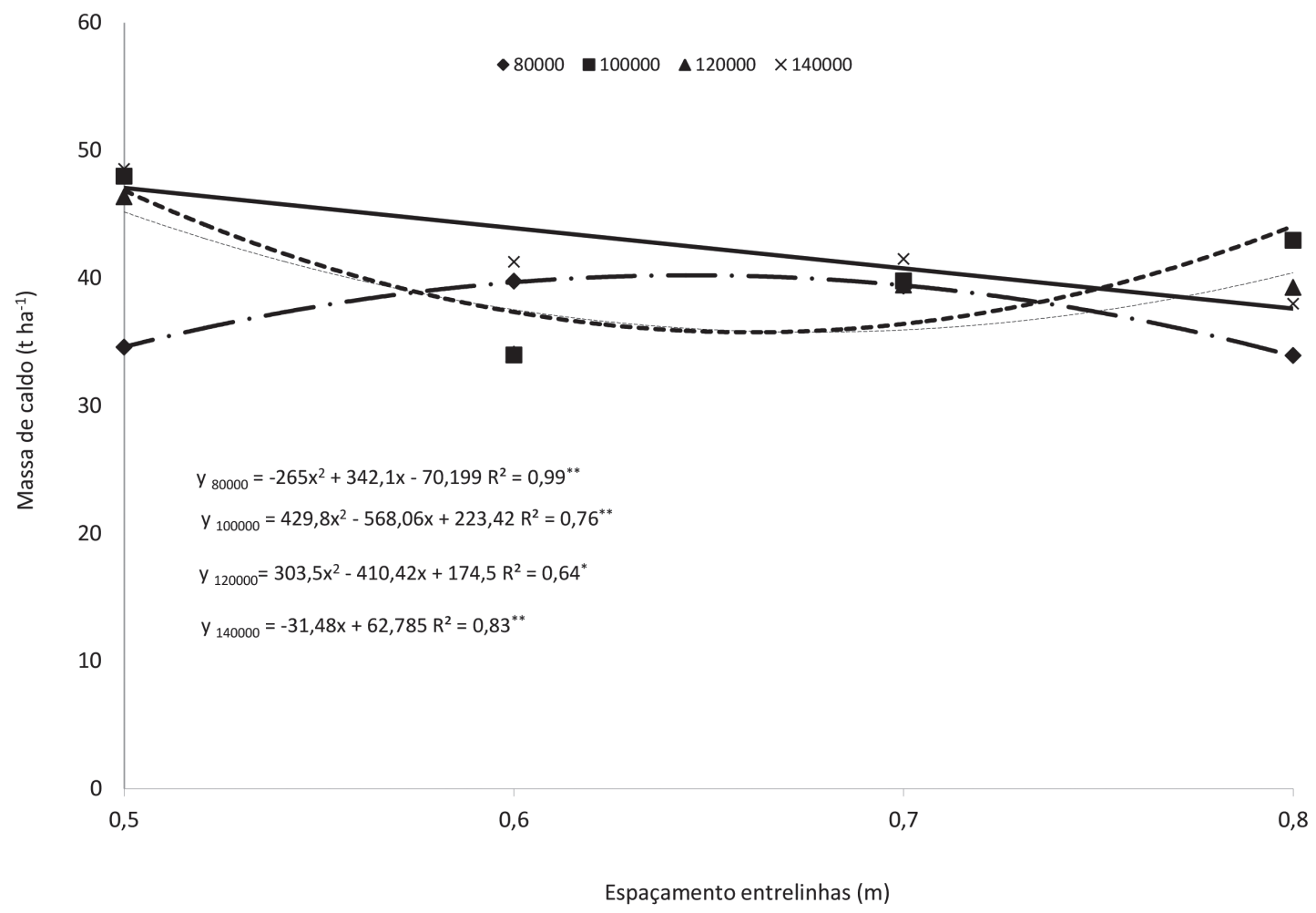

FIGURA 2. Massa de caldo da cultivar de sorgo BRS 505 (Sorghum bicolor), plantada em Patos de Minas, MG, em função do espaçamento entrelinhas. 
$0,95^{* *}$, onde $\mathrm{X}=$ população de plantas $\left(\right.$ plantas ha $\left.\left.^{-1}\right)\right]$. Snider et al. (2011), avaliando plantios de sorgo com diferentes espaçamentos entrelinhas e com diferentes densidades de semeadura, não encontraram diferença significativa para diâmetro de colmos entre os tratamentos em dois locais. Entretanto, em uma terceira área experimental, plantios de sorgo com baixa taxa de semeadura resultaram em plantas com diâmetros maiores.

Altas densidades podem ter provocado aumento na competição intraespecífica por luz, água e nutrientes, proporcionando redução do diâmetro do colmo das plantas. A redução do diâmetro pode tornar as plantas mais sensíveis ao acamamento e ao quebramento, afetando negativamente a colheita da cultura. Avaliando cultivares de sorgo sacarino em Santa Maria, RS, Marchezan \& Silva (1984) encontraram acamamento em plantas de sorgo com diâmetros de $12,3 \mathrm{~mm}$ a $18,0 \mathrm{~mm}$, o mesmo não ocorrendo no presente estudo.

Houve redução do ${ }^{\circ}$ Brix do caldo com o aumento da população de plantas, segundo a equação $\mathrm{Y}$ $=-2 \mathrm{E}-05 \mathrm{X}+17,645\left[\mathrm{R}^{2}=0,82^{* *}\right.$, onde $\mathrm{X}=$ população de plantas (plantas ha-1)], e do espaçamento entrelinhas, segundo a equação $\mathrm{Y}=-4,49 \mathrm{X}+17,862\left[\mathrm{R}^{2}=\right.$ $0,94^{* *}$, onde $\mathrm{X}=$ espaçamento entrelinhas (m)]. Os valores encontrados entre as diferentes combinações estudadas variaram entre 14,27 e 16,05. Estes valores foram menores do que os encontrados para a mesma cultivar em Nova Porteirinha, MG - 20,2 ${ }^{\circ}$ Brix (Souza et al., 2011) e em Santa Maria, RS - 18,2 ${ }^{\circ}$ Brix (Marchezan \& Silva, 1984). De acordo com Prasad et al. (2007), o estágio ideal de colheita é quando o caldo apresenta de 15,5 a $16,5^{\circ}$ Brix, sendo este grau importante para obter um caldo com alta qualidade de fermentação e, consequentemente, maximizar a produção de etanol por hectare. Outros autores aconse- lham médias mais altas de Brix (Almodares \& Hadi, 2009).

O Brix é um termo que representa uma aproximação do conteúdo de sólidos totais, tendo correlação significativa e positiva com a concentração total de açúcar (Teetor et al., 2011) e é diretamente influenciado pela taxa fotossintética das plantas. $\mathrm{O}$ aumento de densidade de semeadura ou o aumento do espaçamento de entrelinha, este último associado ao incremento do número de plantas por linha, pode ter proporcionado maior competição intraespecífica, afetando diretamente a fotossíntese das plantas e, consequentemente, a produção de sólidos solúveis totais.

Para a cultivar BRS 505, o perfilhamento de plantas de sorgo sacarino foi estimulado com o adensamento da linha de plantio, sendo que menores espaçamentos entrelinhas resultaram em maior número de perfilhos por planta $\left[\mathrm{Y}=-0,81 \mathrm{X}+0,7448, \mathrm{R}^{2}=0,82^{* *}\right.$, onde $\mathrm{X}=$ espaçamento entrelinhas $(\mathrm{m})]$. O número de perfilhos variou de 0,10 a 0,34 por planta, contribuindo para o aumento da produção de massa fresca total.

\section{Experimento II - Sete Lagoas, MG}

Os resultados da análise de variância do experimento conduzido em Sete Lagoas, MG, utilizando a cultivar CMSXS 647, demonstraram que houve efeito isolado altamente significativo do espaçamento entrelinhas para a altura de planta, massa fresca total, massa fresca da panícula. Também houve efeito altamente significativo da população de plantas sobre a massa fresca de folhas, a massa fresca de panícula e a massa fresca total. Houve ainda interação altamente significativa entre espaçamento e população de plantas para a massa fresca do colmo e a massa do caldo, porém não houve efeito significativo dos fatores estudados para o diâmetro do colmo, o número de folhas e o Brix do caldo, apresentando valores médios de 
17,1 $\mathrm{mm}$ de diâmetro, 10,4 folhas e $16,55^{\circ}$ Brix, respectivamente. Em nenhum tratamento foi observada a presença de perfilhos.

A cultivar CMSXS 647 apresentou comportamento decrescente de altura com o aumento do espaçamento entrelinhas, a partir de $0,65 \mathrm{~m}$, de acordo com a equação $\mathrm{Y}=-5,17 \mathrm{X}^{2}+6,77 \mathrm{X}+0,111\left[\mathrm{R}^{2}=\right.$ $0,99^{* *}$, onde $\mathrm{X}=$ espaçamento entrelinhas (m)]. Para o espaçamento de $0,5 \mathrm{~m}$ e $0,6 \mathrm{~m}$ entrelinhas, as plantas atingiram 2,20 m e 2,31 m, respectivamente. Snider et al. (2012), trabalhando com diferentes populações e espaçamentos entrelinhas, também verificaram diferença estatística em altura de cultivares de sorgo sacarino, influenciada pelo espaçamento, mas não influenciada pelo número de plantas.

A menor altura das plantas alcançada no maior espaçamento pode ser atribuída, sobretudo, à melhor interceptação de luz neste arranjo de semeadura, diminuindo, assim, a competição por este fator de produção. A competição por luz é uma das modalidades de interferência que provoca maior impacto sobre o crescimento nas plantas, pois restringe a fonte predominante de energia aos processos metabólicos envolvidos no crescimento e no desenvolvimento do vegetal.

A produção de massa fresca total por hectare reduziu com o aumento do espaçamento entrelinhas (Figura 3). Houve queda de 10,63\%, passando de $58,37 \mathrm{t} \mathrm{ha}^{-1}$ de massa fresca total no espaçamento de $0,5 \mathrm{~m}$ para $52,18 \mathrm{t} \mathrm{ha}^{-1}$, com $0,8 \mathrm{~m}$. Este resultado corrobora com o encontrado para a cultivar BRS 505 semeada em Patos de Minas, demonstrando que o sorgo sacarino não tem grande capacidade de compensação na produtividade de biomassa total com alteração no número de linhas cultivadas por hectare. Redução na biomassa, com o aumento do espaçamento entrelinhas, também foi encontrada por outros autores (Albuquerque et al., 2010; Zegada-Lizarazu \& Monti, 2012).

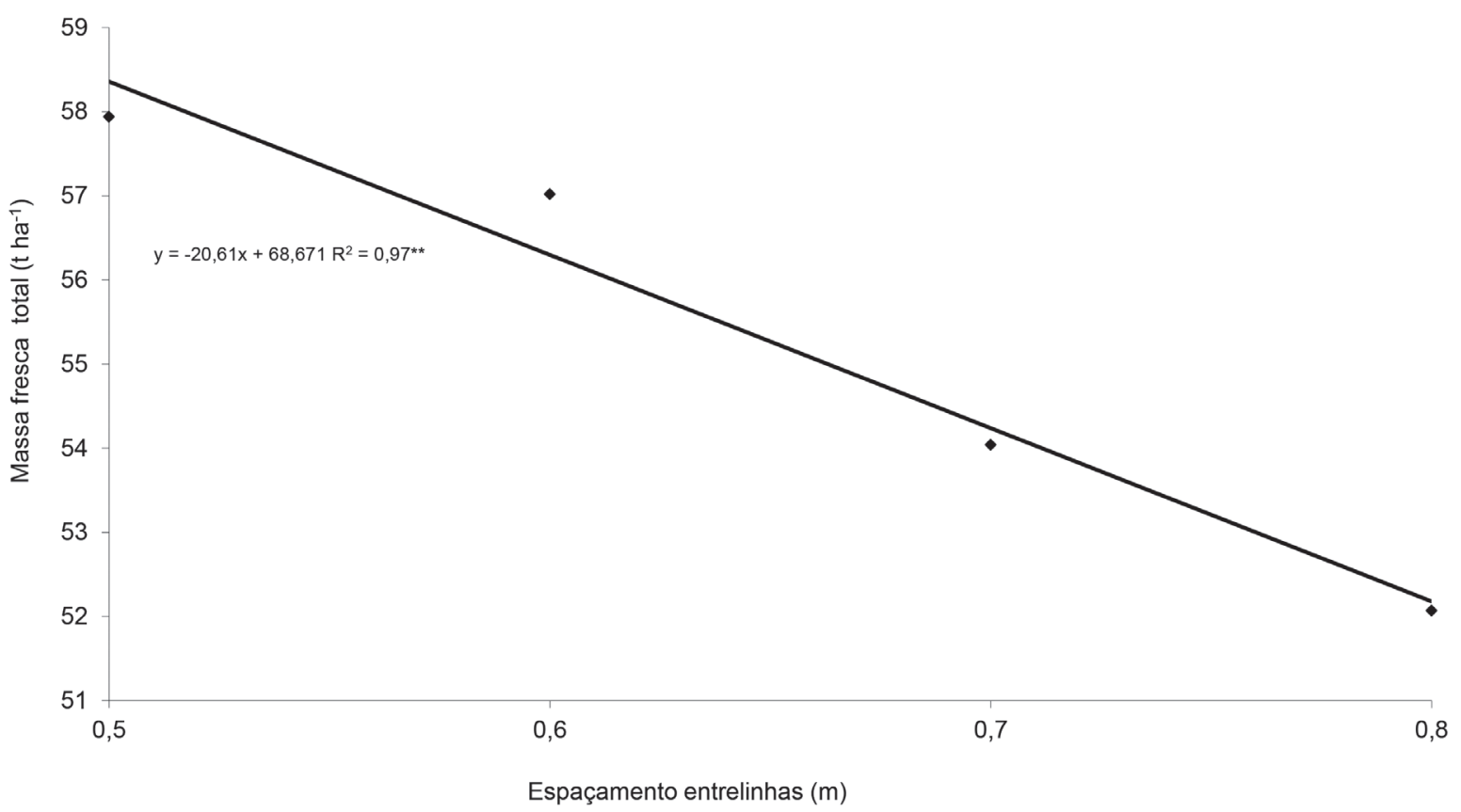

FIGURA 3. Massa fresca total da cultivar de sorgo CMSXS 647 (Sorghum bicolor), plantada em Sete Lagoas, MG, em função do espaçamento entrelinhas. 
Já quando se analisou a produção de massa fresca total em função da densidade de semeadura, observou-se que o incremento de 60.000 plantas ha ${ }^{-1}$ aumentou a produtividade em $6 \mathrm{t} \mathrm{ha}^{-1}$, alcançando $58,57 \mathrm{t} \mathrm{ha}^{-1}$ no stand de 140 mil plantas por hectare (Figura 4). Avaliando a mesma cultivar, Souza et al. (2011) encontraram produção de massa verde variando entre 46,00 e $67,81 \mathrm{t} \mathrm{ha}^{-1}$, plantada no outono e no verão, respectivamente. Han et al. (2012) e Habyarimana et al. (2004) também verificaram aumentos na massa fresca total com o aumento do número de plantas.

A produtividade de massa fresca dos colmos (sem folhas) foi reduzida linearmente com a elevação do espaçamento (Figura 5), mostrando comportamento similar ao que foi observado para a massa fresca total, com menores espaçamentos apresentando maiores produtividades. Porém, o aumento da densidade de semeadura não ocasionou diferença significativa na produtividade de massa fresca de colmo, exceção à densidade de 80.000 plantas ha ${ }^{-1}$ no espaçamento $0,5 \mathrm{~m}$, que apresentou menor rendimento. As maiores produtividades, de 52,84 $\mathrm{t} \mathrm{ha}^{-1}, 53,49 \mathrm{t} \mathrm{ha}^{-1}$ e 53,86 $\mathrm{t} \mathrm{ha}^{-1}$, nos estandes de 100 mil, 120 mil e 140 mil plantas $\mathrm{ha}^{-1}$, respectivamente, foram encontradas no espaçamento 0,5 m entrelinhas. Estas produtividades foram maiores do que as encontradas por Marchezan \& Silva (1984), estudando dez cultivares de sorgo sacarino, que obtiveram uma média de $34,78 \mathrm{t} \mathrm{ha}^{-1}$ de massa verde de colmos.

Por outro lado, a massa fresca de folhas aumentou em função do número de plantas por hectare, conforme a equação $\mathrm{Y}=6 \mathrm{E}-05 \mathrm{X}+0,065\left[\mathrm{R}^{2}=0,98^{* *}\right.$, onde $\mathrm{X}=$ população de plantas (plantas ha-1)]. Embora responsável pela fotossíntese e pela produção de assimilados, esta maior massa das folhas não se reverteu em aumento da massa do caldo ou em diferenças significativas de Brix.

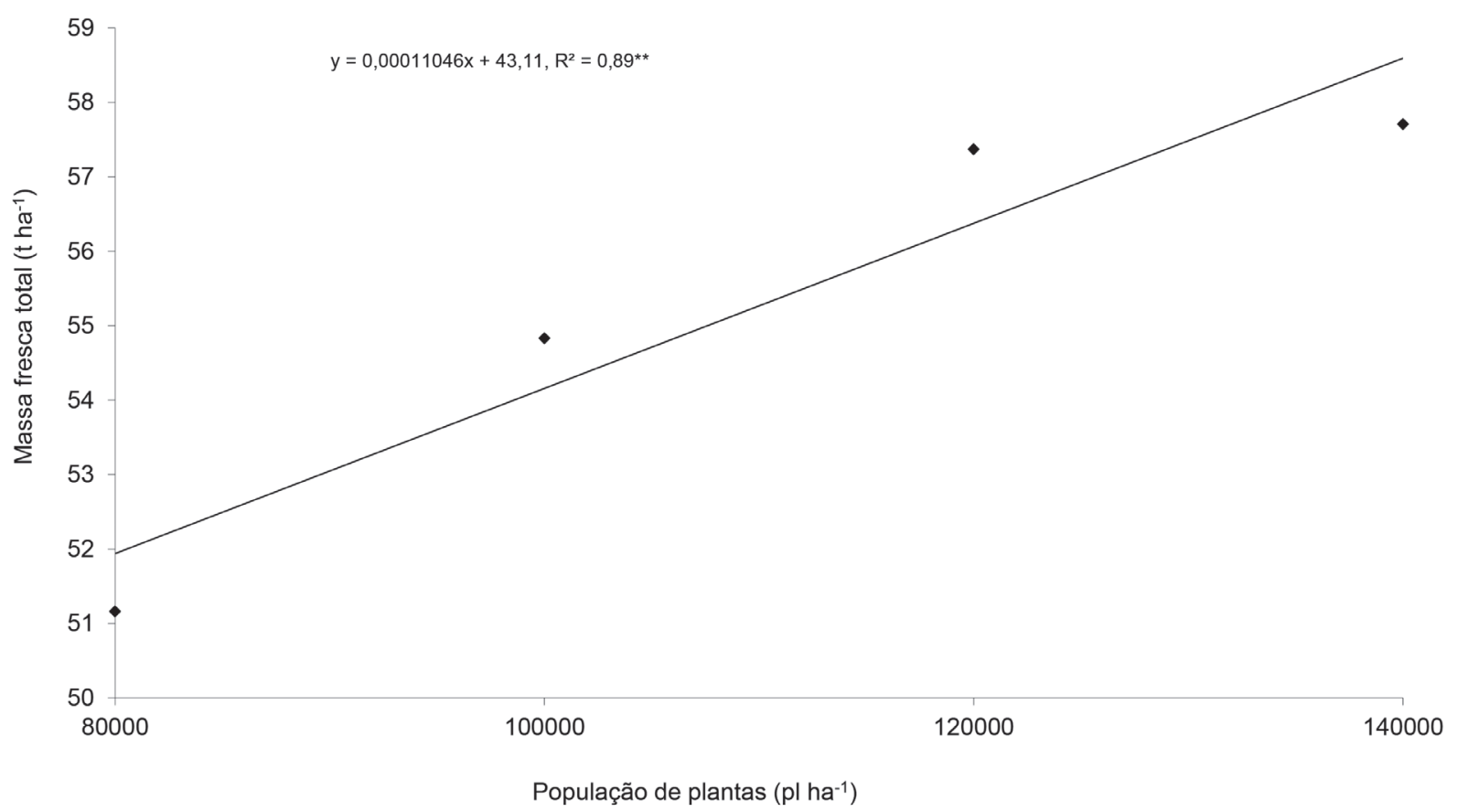

FIGURA 4. Massa fresca total da cultivar de sorgo CMSXS 647 (Sorghum bicolor), plantada em Sete Lagoas, MG, em função da população de plantas. 


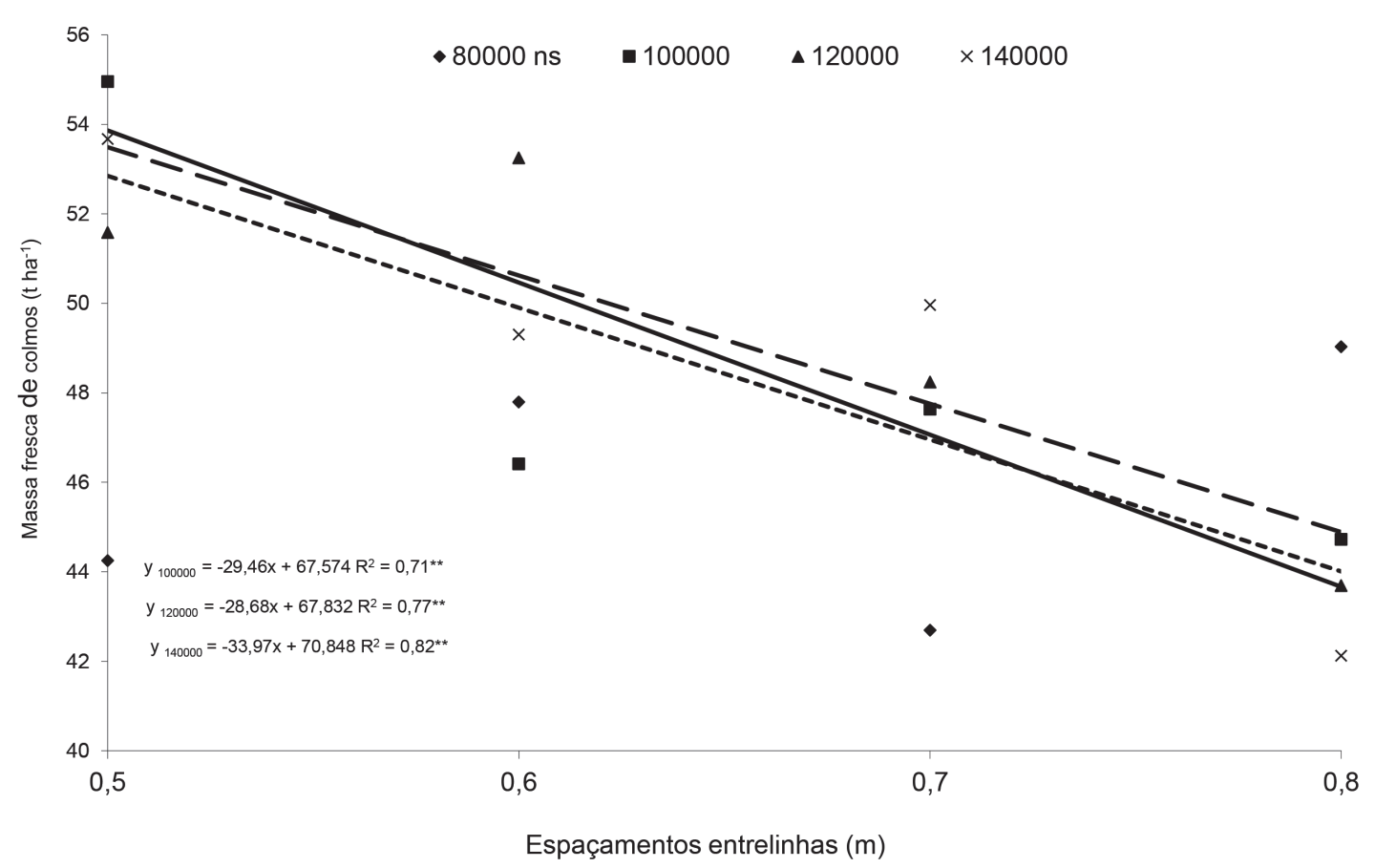

FIGURA 5. Massa fresca de colmos da cultivar de sorgo CMSXS 647 (Sorghum bicolor), plantada em Sete Lagoas, MG, em função do espaçamento entrelinhas.

Não foi observado efeito significativo da população de plantas sobre a massa fresca de colmos, sugerindo que o incremento da massa fresca total com o aumento da população de plantas foi decorrente do aumento da massa fresca de folhas. Assim, como anteriormente citado, o espaçamento entrelinhas foi mais efetivo em influenciar a produtividade de colmos.

A mesma tendência apresentada para produtividade de massa de colmos foi encontrada para a massa de caldo, que apresentou queda linear de produtividade com o aumento dos espaçamentos utilizados (Figura 6). Isto demonstra que, para a cultivar CMSXS 647, incrementos na população de plantas não geram elevações na produção de caldo e, consequentemente, de etanol, por área cultivada, principalmente para populações superiores a 100.000 plantas $\mathrm{ha}^{-1}$. As maiores produtividades de caldo foram obtidas com a combinação do espaçamento entrelinhas de $0,5 \mathrm{~m}$ e populações de plantas de 100 mil, 120 mil ou 140 mil plantas ha-1 ${ }^{-1}$, sugerindo que a redução no espaçamento de entrelinhas é um fator de elevada importância para a manutenção de altas produtividades, principalmente quando se considera que o Brix do caldo não foi afetado pela alteração no arranjo de plantas, como foi o caso do experimento realizado em Sete Lagoas, MG.

Teetor et al. (2011), avaliando cultivares de sorgo sacarino, sugerem que menores quantidades de plantas por área podem ser favoráveis ao aumento de atributos de colheita, como peso das hastes e massa de caldo, devido à menor competição pelos recursos e à alta interceptação de luz, entre outros. Entretanto, o efeito sobre a concentração de açúcar ainda não é claro. Han et al. (2012) discutem que o aumento da absorção da radiação solar no início do crescimento das plantas, através do aumento no número de plantas e 


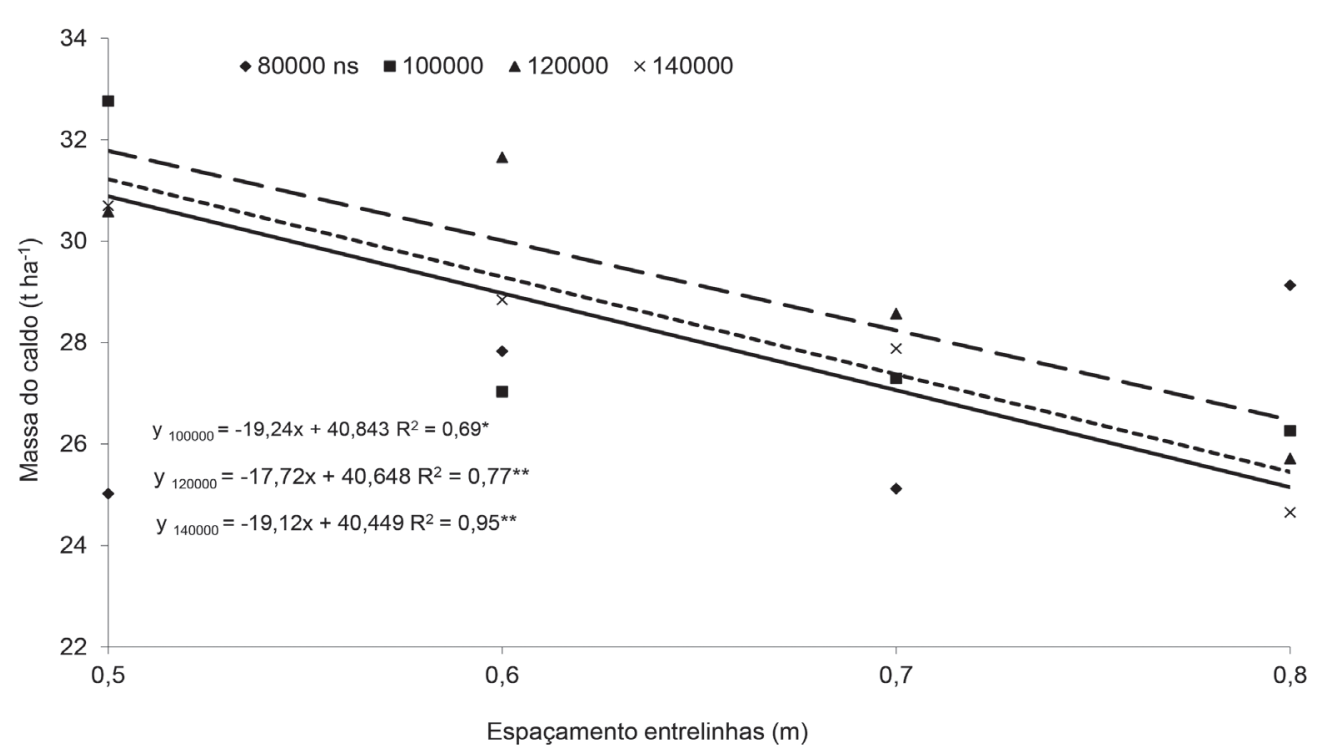

FIGURA 6. Massa do caldo da cultivar de sorgo CMSXS 647 (Sorghum bicolor), plantada em Sete Lagoas, MG, em função do espaçamento entrelinhas.

da maior uniformidade de distribuição delas na área, pode favorecer, mais tarde, o aumento da eficiência no uso da energia pelas plantas de sorgo sacarino, revertendo em aumento de produtividade.

Embora não influenciado pelos fatores estudados, o valor médio de ${ }^{\circ}$ Brix foi de 16,55, próximo ao valor encontrado por Souza et al. (2011) para a mesma cultivar, de $15,2{ }^{\circ}$ Brix, plantada na mesma época, no Semiárido mineiro e dentro da faixa considerada desejável por Davila-Gomes et al. (2011).

Observou-se redução da massa de panícula em função tanto do aumento do espaçamento entrelinhas $\left[\mathrm{Y}=-44 \mathrm{X}+101,04, \mathrm{R}^{2}=0,88^{* *}\right.$, onde $\mathrm{X}=$ espaçamento entrelinhas (m)], como do aumento da população de plantas $\left[\mathrm{Y}=-0,0002 \mathrm{X}+94,254, \mathrm{R}^{2}=\right.$ $0,54^{*}$, onde $\mathrm{X}=$ população de plantas $($ plantas ha-1)].

\section{Conclusão}

O arranjo de plantas, através da alteração no espaçamento entrelinhas ou na população de plantas, influencia o crescimento das plantas de sorgo sacarino, resultando em maiores produtividades de caldo quando cultivadas em menores espaçamentos de entrelinhas e maiores populações de plantas. Para a massa de caldo, a redução no espaçamento entrelinhas proporciona maiores incrementos na produção em comparação à elevação na população de plantas e eleva o Brix do caldo. As cultivares estudadas apresentaram, em geral, semelhante padrão de resposta às variações no espaçamento entrelinhas e na densidade de plantas.

\section{Agradecimentos}

Os autores agradecem à Epamig Patos de Minas, pelo apoio na condução dos experimentos realizados na unidade de pesquisa regional, e à Comunidade Econômica Europeia, pelos recursos disponibilizados através do Projeto Sweet Fuel da Embrapa Milho e Sorgo, liderado pelo pesquisador Dr. Robert Eugene Schaffert. 


\section{Referências}

ALBUQUeRQUe, C. J. B.; PARRELA, R. A. C.; TARDIN, F. D.; BRANT, R. S.; SIMÕES, D. A.; FONSECA JÚNIOR, W. B.; OLIVEIRA R. M.; JESUS, K. M. Potencial forrageiro de cultivares de sorgo sacarino em diferentes arranjos de plantas e localidades de Minas Gerais. In: CONGRESSO NACIONAL DE MILHO E SORGO, 28.; SIMPÓSIO BRASILEIRO SOBRE LAGARTA DO CARTUCHO, 4., 2010, Goiânia. Potencialidades, desafios e sustentabilidade: resumos expandidos. Goiânia: ABMS, 2010. 1 CD-ROM.

ALMODARES, A.; HADI, M. R. Production of bioethanol from sweet sorghum: a review. African Journal of Agricultural Research, Washington, v. 4, n. 9, p. 772-780, 2009.

ALVAREZ V., V. H.; NOVAIS, R. F.; BARROS, N. F.; CANTARUTTI, R. B.; LOPES, A. S. Interpretação dos resultados das análises de solos. In: RIBEIRO, A. C.; GUIMARAES, P. T. G.; ALVAREZ V., V. H. (Ed.). Recomendação para o uso de corretivos e fertilizantes em Minas Gerais: 5a. aproximação. Viçosa, MG: Comissão de Fertilidade do Solo do Estado de Minas Gerais, 1999. p. 25-32.

AMADUCCI, S.; MONTI, A.; VENTURI, G. Non-structural carbohydrates and fibre components in sweet and fibre sorghum as affected by low and normal input techniques. Industrial Crops Products, Tucson, v. 20, n. 1, p. 111-118, 2004.

CONAB. Acompanhamento da safra brasileira: cana-de-açúcar, safra 2011/2012: terceiro levantamento: dezembro/2011. Disponível em: <http:// www.conab.gov.br/OlalaCMS/uploads/arquivos/11_12_08_11_00_54_08.pdf >.Acesso em: 15 fev. 2012.

COWLEY, O. H.; SMITH, B. S. Sweet sorghum as a potential sugar crop in south Texas. In: CONGRESS
OF THE INTERNATIONAL SOCIETY OF SUGAR CANE TECHNOLOGY, 14., 1971, New Orleans. Proceedings. New Orleans: [s.n.], 1972. p. 628-633.

DAVILA-GOMEZ, F. J.; CHUCK-HERNANDEZ, C.; PEREZ-CARRILLO, E.; ROONEY, W. L.; SERNA-SALDIVAR, S. O. Evaluation of bioethanol production from five different varieties of sweet and forage sorghums (Sorghum bicolor (L) Moench). Industrial Crops and Products, Tucson, v. 33, p. 611-616, 2011. HABYARIMANA, E.; BONARDI, P.; LAURETI, D.; DI BARI, V.; COSENTINO, S.; LORENZONI, C. Multilocational evaluation of biomass sorghum hybrids under two stand densities and variable water supply in Italy. Industrial Crops and Products, Tucson, v. 20, p. 3-9, 2004.

HAN, K. J.; PITMAN, W. D.; ALISON, M. W.; HARRELL, D. L.; VIATOR, H. P.; McCORMICK, M. E.; GRAVOIS, K. A.; KIM, M.; DAY, D. F. Agronomic considerations for sweet sorghum biofuel production in the South-Central USA. Bioenergy Research, New York, v. 5, p. 748-758, 2012.

MARCHEZAN, E.; SILVA, M. I. Avaliação de cultivares de sorgo em Santa Maria, RS. Revista do Centro de Ciências Rurais, Santa Maria, v. 14, n. 3/4, p. 161172,1984

PRASAD, S.; SINGH, A.; JAIN, N.; JOSHI, H. C. Ethanol production from sweet sorghum syrup for utilization as automotive fuel in India. Energy and Fuels, Washington, v. 21, p. 2415-2420, 2007.

RATNAVATHI, C. V.; CHAKRAVARTHY, S. K.; KOMALA, V. V.; CHAVAN, U. D.; PATIL, J. V. Sweet sorghum as feedstock for biofuel production: a review. Sugar Tech, Heidelberg v. 13, n. 4., p. 399-407, 2011. RODRIGUES, E. F.; LEITE, I. C. Crescimento de genótipos de sorgo plantados nos sentidos norte-sul e leste-oeste. Pesquisa Agropecuária Brasileira, Brasília, DF, v. 34, n. 2, p. 173-179, 1999. 
SNIDER, J. L.; RAPER, R. L.; SCHWAB, E. B. The effect of row spacing and seeding rate on biomass production and plant stand characteristics of non-irrigated photoperiod-sensitive sorghum (Sorghum bicolor (L.) Moench). Industrial Crops and Products, Tucson, v. 37, p. 527-535, 2012.

SOUZA, V. F.; PARRELLA, R. A.; PORTUGAL, A. F.; TARDIN, F. D.; DURÃES, N. N. L.; SCHAFFERT, R. E. Desempenho de cultivares de sorgo sacarino em duas épocas de plantio no norte de Minas Gerais visando a produção de etanol. In: CONGRESSO BRASILEIRO DE MELHORAMENTO DE PLANTAS, 6. 2011, Búzios. Panorama atual e perspectivas do melhoramento de plantas no Brasil. [Búzios]: SBMP, 2011. 1 CD-ROM

TEETOR, V. H.; DUCLOS, D. V.; WITTENBERG, E. T.; YOUNG, K. M.; CHAWHUAYMAK, J.; RILEY, M. R.; RAY, D. T. Effects of planting date on sugar and ethanol yield of sweet sorghum grown in Arizona. Industrial Crops and Products, Tucson, v. 34, p. 1293-1300, 2011. TEIXEIRA, C. G.; JARDINE, J. G.; NICOLELLA, G.; ZARONI, M. H. Influência da época de corte sobre o teor de açúcares de colmos de sorgo sacarino. Pesquisa Agropecuária Brasileira, Brasília, DF, v. 34, n. 9, p. 1601-1606, 1999.

TEIXEIRA, C. G.; JARDINE, J. G.; DARCY, A. Utilização do sorgo sacarino como matéria-prima complementar à cana-de-açúcar para obtenção de etanol em microdestilaria. Ciência e Tecnologia de Alimentos, Campinas, v. 17, n. 3, p. 248-251, 1997.

VASILAKOLOGLOU, I.; DHIMA, K.; KARAGIAMNNIDIS, N.; GATSIS, T. Sweet sorghum productivity for biofuels under increased soil salinity and reduced irrigation. Field Crops Research, Amsterdam, v. 120, p. 38-46, 2011.

ZEGADA-LIZARAZU, W.; MONTI, A. Are we ready to cultivate sweet sorghum as a bioenergy feedstock? A review on field management practices. Biomass and Bioenergy, Oxford, v. 40, p. 1-12, 2012.

ZHAO, Y. A.; DOLAT A.; STEINBERGER, Y.; WANGA, X.; OSMAN, A.; XIE, G. H. Biomass yield and changes in chemical composition of sweet sorghum cultivars grown for biofuel. Field Crops Research, Amsterdam, v. 111, p. 55-64, 2009. 